\title{
Shear deformation and fracture of human cortical bone
}

Tengteng Tang ${ }^{\text {ad }}$, Vincent Ebacher ${ }^{\text {ad }}$, Peter Cripton ${ }^{\text {bd }}$, Pierre Guy ${ }^{\text {cd }}$, Heather McKay ${ }^{\text {cd }}$, Rizhi Wang $^{\text {ad, } *}$

${ }^{a}$ Department of Materials Engineering, University of British Columbia, Vancouver, BC, Canada.

${ }^{b}$ Department of Mechanical Engineering, University of British Columbia, Vancouver, BC, Canada.

${ }^{\mathrm{c}}$ Department of Orthopaedics, University of British Columbia, Vancouver, BC, Canada.

${ }^{\mathrm{d}}$ Centre for Hip Health and Mobility, Vancouver, BC, Canada.

*Correspondence address:

Dr. Rizhi Wang

Department of Materials Engineering

University of British Columbia

309-6350 Stores Road

Vancouver, BC V6T 1 Z4

Canada

Tel: 1-604-822-9752

Fax: 1-604-822-3619

Email: rzwang@mail.ubc.ca

DOI: 10.1016/j.bone.2014.10.001.

(C) 2015. This manuscript version is made available under the CC-BY-NC-ND 4.0 license https://

creativecommons.org/licenses/by-nc-nd/4.0/ 


\title{
Shear Deformation and Fracture of Human Cortical Bone
}

\begin{abstract}
Bone can be viewed as a nano-fibrous composite with complex hierarchical structures. Its deformation and fracture behavior depend on both the local structure and the type of stress applied. In contrast to the extensive studies on bone fracture under compression and tension, there is a lack of knowledge on the fracture process under shear, a stress state often exists in hip fracture. This study investigated the mechanical behavior of human cortical bone under shear, with the focus on the relation between the fracture pattern and the microstructure. Iosipescu shear tests were performed on notched rectangular bar specimens made from human cortical bone. They were prepared at different angles (i.e. $0^{\circ}, 30^{\circ}, 60^{\circ}$ and $90^{\circ}$ ) with respect to the long axis of the femoral shaft. The results showed that human cortical bone behaved as an anisotropic material under shear with the highest shear strength ( 50 MPa) obtained when shearing perpendicular to the Haversian systems or secondary osteons. Digital image correlation (DIC) analysis found that shear strain concentration bands had a close association with long bone axis with an average deviation of $11.8^{\circ}$ to $18.5^{\circ}$. The fracture pattern was also greatly affected by the structure with the crack path generally following the direction of the long axes of osteons. More importantly, we observed unique peripheral arc-shaped microcracks within osteons, using laser scanning confocal microscopy (LSCM). They were generally long cracks that developed within a lamella without crossing the boundaries. This microcracking pattern clearly differed from that created under either compressive or tensile stress: these arc-shaped microcracks tended to be located away from the Haversian canal in early-stage damaged osteons, with $\sim 70 \%$ developing in the outer third osteonal wall. Further study by second harmonic generation (SHG) and twophoton excitation fluorescence (TPEF) microscopy revealed a strong influence of the organization of collagen fibrils on shear microcracking. This study concluded that shear-induced microcracking of human cortical bone follows a unique pattern that is governed by the lamellar structure of the osteons.
\end{abstract}

Keywords: Bone fracture; Shear; Microcracking; Digital image correlation; Second harmonic generation. 


\section{Introduction}

Bone fracture, especially hip fracture is a serious and costly public health problem. Approximately 1.5 million hip fractures occur each year worldwide, and the prevalence increases to 2.6 million by 2025 and a staggering 4.5 million by 2050 [1]. Hip fractures are also associated with huge economic burden and increased risk of death, with $8.4 \%$ to $36 \%$ mortality within 1 year after the fracture incident [2]. From a materials point of view, hip fracture is the combined result of a fragile bone structure and the complicated stress state of tension, compression, and shear caused by external impact. It is essential to better understand how bone fracture under different stress states and how this relates to bone's hierarchical structures as a means to improve clinical risk assessment and to contribute to the development of solutions to prevent hip fracture.

Prior to fracture, human cortical bone exhibits significant inelastic deformation, an important characteristic that directly contributes to bone's high resistance to fracture [3]. Inelastic deformation is accompanied by the formation and development of microcracks [3, 4]. The pattern of microcracks strongly depends on the local stress. In tension, densely distributed wavy microcracks form roughly normal to the direction of the stress with crack length around 2$10 \mu \mathrm{m}$ [4-6]. In contrast, compressive microcracks are straight and relatively longer; forming a typical cross-hatched pattern, orienting at approximately $27^{\circ}-40^{\circ}$ to the long axis of the bone [3, 7-9]. If such a specific relation between the microcracking pattern and the applied stress is confirmed for all stress states, one could potentially use the microcracks as "finger prints" to reconstruct the at-fracture stress state of a complicated clinical hip fracture. Most previous studies of bone failure at the material level have focused on tension and compression while far fewer studies have focused on shear-induced microcracking [10-12], despite the fact that buckling-induced shear between lamellae is likely to be involved in femoral neck fractures [13, 14]. Ascenzi et al. were among the earliest to investigate the shearing properties of single osteons [15]. Their work on single osteons under compression [16] showed that the microcracks created by compression-induced shearing formed an angle of roughly $30^{\circ}-35^{\circ}$ with the axis of the osteon. With detailed electron microscopic study, they have found circular cracks in the osteons having alternating fiber orientations in adjacent layers [17]. Later torsional studies showed that damage appeared within interstitial bone, Haversian systems, and along cement lines [18, 19]. However, since torsional tests generate non-uniform shear in the specimens, they are not suitable for 
studying bone tissue behavior in pure shear mode [20, 21]. More importantly, how shear induces microcracks within or between lamellae at the cortical bone level is still unknown. Our previous study on human cortical bone under compression revealed a clear sub-lamellar cross-hatched pattern, indicating an interfibrillar cracking nature [22]. We thus hypothesized that human cortical bone under shear deformed through intralamellar and interfibril microcracking.

Therefore, the purpose of this study was to investigate the shear-induced deformation and microcracking processes in human cortical bone and their relationship with bone's hierarchical structures, especially the collagen fibril orientation. Our specific objectives were to: 1. identify unique microcracking pattern under shear; 2. quantitatively define the shear-induced microcracks in osteons at the osteonal-interstitial level; and 3. specifically link the shear-induced microcracks to the lamellar structure at the micro-level.

\section{Materials and Methods}

\section{Study Design and General Approach}

We conducted mechanical testing followed by microscopic examination on the transversal plane (i.e. perpendicular to the long axes of osteons) to address our objectives. Specifically, we combined Iosipescu shear tests (ASTM standard D 5379) with digital image correlation (DIC) for in-plane strain analysis, and fluorescence staining and laser scanning confocal microscopy (LSCM) for microcrack imaging. To access the interactions between bone's hierarchical structures and microcracking and deformation process, we integrated second harmonic generation (SHG) [23] and two-photon excitation fluorescence (TPEF) [24] imaging techniques to relate the unique microcracking patterns to bone structure at the sub-lamellar level. Although SHG and TPEF have been used for biological and medical imaging for almost two decades, their potential has not yet been fully explored for imaging bone and microcracking [25]. We provide more detailed methods below.

\section{Specimens Preparation}

We obtained eight freshly frozen human cadaver femurs (six males, two females, age range: 62-79) without reported metabolic bone tissue conditions from LifeLegacy Foundation. 
All bone tissues were kept frozen at $-20^{\circ} \mathrm{C}$ until specimen preparation. The study was approved by the Clinical Research Ethics Review Board at the University of British Columbia.

Twenty-nine specimens in total were subjected to shear testing (ten $0^{\circ}$, nine $30^{\circ}$, five $60^{\circ}$ and five $90^{\circ}$ orientation, respectively). The shear specimens were obtained from the cortex of the diaphysis region. Two cuts, each perpendicular to the femoral shaft's long axis, were made at $27 \%$ and $48 \%$ from the proximal end of each femur with a band saw. The medial quadrant of each bone cylinder thus obtained was then sectioned into four different orientation bar specimens (Figure 1), i.e. oriented at $0^{\circ}, 30^{\circ}, 60^{\circ}$ and $90^{\circ}$ to the long bone axis, and thus the long axes of majority osteons, by a low speed diamond saw (IsoMet 1000, Buehler Ltd, ON, Canada). The four sides of each specimen aligned either parallel or normal to the periosteal surfaces of femoral shaft. Rough machining was performed using a mini-milling machine (Sherline, model 5400, Vista, CA, USA). The specimens were subsequently manually grounded, using a series of carbide grinding papers (Buehler Buehler Ltd, ON, Canada), and polished with diamond suspension staring with $6 \mu \mathrm{m}$ and ending with $1 \mu \mathrm{m}$ (Leco Co., St. Joseph, MI, USA). The final specimens were $20 \mathrm{~mm}$ long, $5 \mathrm{~mm}$ wide and $3 \mathrm{~mm}$ thick, with the front and back surfaces (20 $\mathrm{mm} \times 5 \mathrm{~mm}$ ) parallel to the periosteal surface of femoral shaft. By following the ASTM standard (ASTM D5379/D5379M) [26], two 90 V-shaped notches, approximately 22\% of the depth of the specimen total width, were made with a razor blade at the top and bottom surfaces (i.e. 20 $\mathrm{mm} \times 3 \mathrm{~mm}$ ). All the above steps were done under constant water irrigation. After the initial preparation, we assessed bone mineral density $\left(\mathrm{g} / \mathrm{cm}^{3}\right)$ in all specimens using peripheral quantitative computed tomography (pQCT, model XCT 3000, Stratec, Biomedical AG, Germany) with a voxel size of $0.2 \times 0.2 \times 2.3 \mathrm{~mm}$. The entrance dose of the irradiation was 1 $\mu \mathrm{Gy}$ which is extremely low and safe to bone specimens. We further statistically compared for differences relatively to their orientations using Kruskal-Wallis one-way ANOVA test in SPSS with a confidence level of $95 \%$ ( $\mathrm{p}<0.05)$. In addition, we inspected specimens for pre-existing cracks after specimen preparation by xylenol orange (Sigma-Aldrich) staining at a concentration of $0.5 \mathrm{mM}$ [27]. Specimens were wrapped with tissue paper, soaked with phosphate-buffered saline solution (0.1 mol; $\mathrm{pH} 7.6)$, and kept at $-20^{\circ} \mathrm{C}$ in sealed plastic bags until the day of testing.

\section{Shear Testing}


Iosipescu shear tests followed the ASTM standard (D5379/D5379M) [26]. The design of the Iosipescu method eliminates bending moment across the notched section and creates a uniform shear plane between the loading points [28]. The fixture developed by Adams and Walrath [29], known as the 'modified Wyoming fixture' is widely used in composite research laboratories. However, the correct measurement of shear modulus and shear strength of synthetic composite materials are still debatable [30]. Shear modulus $\left(G=\frac{\tau_{x y}}{\gamma_{x y}}\right)$ as measured only applies when the shear stress and strain distribution are uniform at the test section of the specimens. This is difficult to achieve practically when testing anisotropic materials such as bone [31]. To date, only a few studies have applied the Iosipescu shear test to bone [20, 28]. Nevertheless, it is the most mature in-plane shear test that we are aware of. During the test, each specimen was gripped in a customized fixture (Wyoming Test Fixtures, Salt Lake, UT, USA) (Figure 1). All the tests were performed at a cross head speed of $0.2 \mathrm{~mm} / \mathrm{min}$ on an electromechanical testing machine (Minimat 2000, Maple Instruments Ltd., Toronto, ON, Canada) equipped with a $1 \mathrm{kN}$ load cell. All specimens were loaded to final fracture to obtain the full load-displacement curves (i.e. at about $90 \%$ drop from the peak load). Experiments were performed at room temperature $\left(20^{\circ} \mathrm{C}\right)$, and specimens were kept wet during testing. Time, load, and displacement were recorded. Shear strengths were calculated following ASTM standard (D5379/D5379M) for the four orientation groups and statistically compared using Kruskal-Wallis one-way ANOVA test with a confidence level of $95 \%(p<0.05)$. The stiffness of the testing system was calibrated using a 1018 mild steel specimen (shear modulus $\mathrm{G}=80 \mathrm{GPa}$ ).

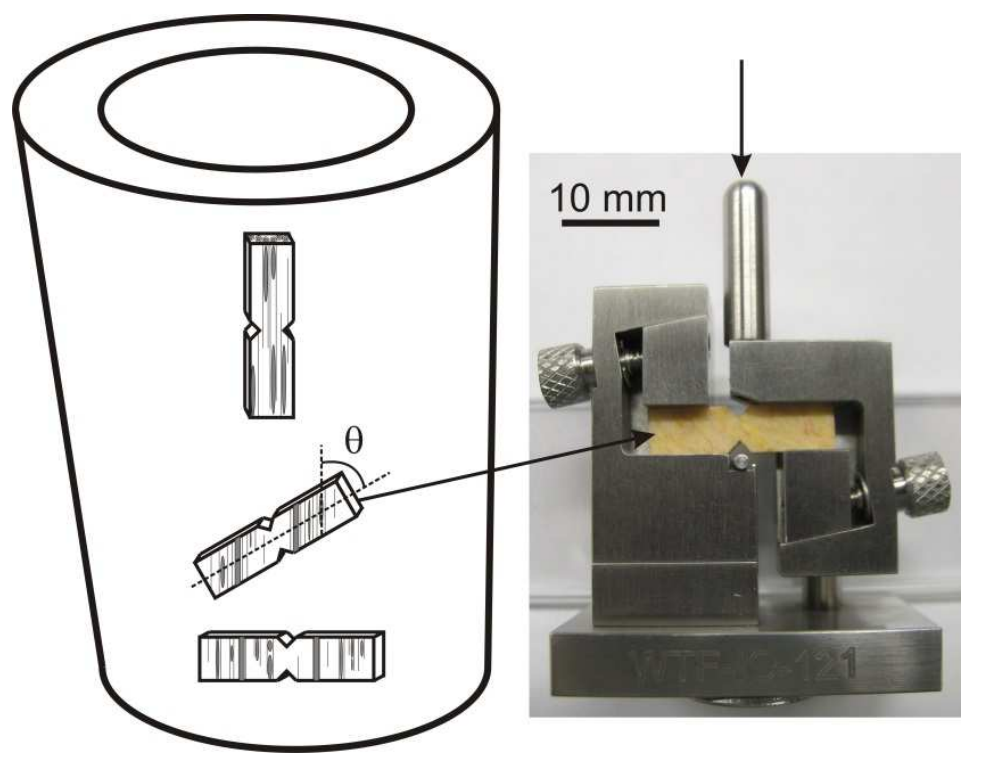


Fig. 1 Schematic illustration of specimens prepared from human femora. Shear specimens were oriented at $0^{\circ}, 30^{\circ}$, $60^{\circ}$ and $90^{\circ}$ with respect to the long axis of femora, which is indicated by the grey lines. A specimen was gripped in the custom made Wyoming Iosipescu fixture with the shear loading indicated by the arrow pointing at the top of the rod.

\section{Digital Image Correlation and Strain Analysis}

In-plane displacement and strain fields were obtained using a digital image correlation (DIC) [32]. This analysis provided information on the deformation process of human cortical bone during shear testing. The intrinsic microstructural features of the polished bone provide high contrast surface patterns, which enables one to use DIC without spraying speckle pattern on specimens' surfaces. All specimens were tested with a gage section (approximately $3.6 \mathrm{~mm}$ x 2.7 $\mathrm{mm}$ ) imaged under an optical microscope (Nikon Eclipse E600) equipped with a digital CCD camera (QImaging QICAM Fast 1349, Surrey, BC, Canada). A sequence of 1392 x 1040 pixels with 8-bits color images was captured under the reflected light for each specimen at the frame rate of 4 frames/s. The images were later selected at regular intervals and processed using the Davis software (Davis LaVision Inc., Ypsilanti, MI, USA) to compute the in-plane displacement fields and finally the in-plane strain fields. The angles of shear strain bands on the surfaces were measured with respect to the bone's long axis with ImageJ software (U. S. NIH, Maryland, USA). During the shear tests, a high-speed video camera (Phantom V12, Vision Research, NJ, USA) equipped with a Nikon $105 \mathrm{~mm}$ macro f2.8 lens (Nikon Co., Tokyo, Japan) was also used to monitor the fracture progress at the frame rate of 800 frames/s.

The shear stress-strain curves we present are based on the nominal shear stress, following ASTM standard (D5379/D5379M), and engineering shear strain which was calculated based on a rectangular area covering $80 \%$ of the surface between two notches (Figure 3; illustration). The shear moduli were measured from the slope of the initial linear part of the stress-strain curves.

In traditional engineering material study, the concept of stress triaxiality is often used to assess the mechanism of fracture [33]. The value of stress triaxiality shows the loading condition of the testing material: tension, compression or shear. Similar to stress triaxiality concept, strain triaxiality was later deducted [34], and developed to adapt to the in-plane shear test as [35]:

$T R^{*}=\frac{\frac{1}{3}\left(\varepsilon_{1}+\varepsilon_{2}\right)}{\sqrt{\frac{1}{2}\left[\left(\varepsilon_{1}-\varepsilon_{2}\right)^{2}+\varepsilon_{1}{ }^{2}+\varepsilon_{2}{ }^{2}\right]}}$ 
where $\varepsilon_{1}$ and $\varepsilon_{2}$ stands for maximum normal strain and minimum normal strain, respectively. When $T R^{*}$ value is 0 , it corresponds to pure shear. While if tension is more prominent, $T R^{*}$ value should be positive, and $T R^{*}$ should be negative in compression. If the absolute value is much less than $1 / 3$, we assume that shear strain predominates over the gauge section [35].

\section{Microcracking Analysis}

In order to observe the microcracks' morphology, distribution and extent, all specimens were fixed, dehydrated and stained after mechanical tests, following a protocol used by Ebacher et al. [9]. Briefly, the specimens were fixed in $70 \%$ acetone/water solution for 24 hours, followed by dehydration in a graded ethanol series $(80 \%, 90 \%$, and 100\%) for 24 hours per step and finally stained in a filtered saturated solution of fluorescein (Fisher Scientific) dissolved in 70\% ethanol/water overnight. The specimens were then rinsed in $100 \%$ ethanol until the washed ethanol was clear before being air-dried.

After staining, the specimens were examined under a stereomicroscope (Nikon SMZ 1000) to characterize their macroscopic fracture patterns. The angles of the major fracture initiation with respect to the bone's long axis were measured. Optical microscope (Nikon Eclipse E600) using reflected white light and epi-fluorescence light (with excitation at approximately $490 \mathrm{~nm}$ and emission at approximately $525 \mathrm{~nm}$ ) were used for initial microcracks examination. Three specimens from each group were then cut in the plane perpendicular to the bone's long axis to observe the relation between fracture and microstructure in the gauge zone. Further observation was under laser scanning confocal microscope (LSCM; Olympus FluoView FV1000, Olympus Canada Inc.; Leica SP5X, Concord, Ontario, Canada). During the high magnification LSCM imaging process, specimens were immersed in an in-house built chamber filled with pure ethanol (Commercial Alcohols Inc.) to reduce the effects of reflective index difference [22]. The $488 \mathrm{~nm}$ line of a multi-line Argon laser was used for fluorescein excitation, and the emission was at $519 \mathrm{~nm}$. Selected sites of microcracks were further characterized in three dimensions (3D), during which the specimens were typically imaged from the surface down to depths of $15 \mu \mathrm{m}-20 \mu \mathrm{m}$ with a step size of $200 \mathrm{~nm}$. Each series of 12-bit images thus obtained was reviewed, analyzed, and, when necessary, stacked in z-plane (Imaris 7.6.0, Bitplane AG) to obtain microcrack morphology in the third direction. Additionally, in order to verify whether the state of microcracks as seen on the longitudinal surfaces was representative of the damage in the 
bulk of the material, three $0^{\circ}$ specimens were grounded and polished to their centre parallel to the longitudinal surface. Variable pressure scanning electron microscope (SEM; Hitachi S-3000N, Hitachi Ltd., Tokyo, Japan) was also used to show the local bone lamellar structure and detect crack deflections at osteons.

Second harmonic generation (SHG) combined with two-photon excitation fluorescence (TPEF) imaging was used to evaluate the location of microcracks in terms of bone's lamellar structure. This was performed on selected specimens under Olympus Multiphoton Laser Scanning Microscope FV1000 (MPM, Olympus Co., Shinjuku-ku, Tokyo, Japan) with the objective lens at 25X, N.A.=1.05 and 60X, N.A.=1.4. The SHG signal originates from non-linear optical materials possessing a non-centrosymmetric molecular organization such as collagen [36]. The signal intensity depends on the collagen orientation with the strongest signal from the ones lying in the plane. It is well known that lamellar bone is made up of preferentially orientated collagen fibrils organized in lamellae [37]. When imaged under SHG, high contrast image is produced, providing valuable information on the preferred orientation of collagen fibrils in bone. Compared to traditional techniques for imaging bone structure at the micro-scale, SHG has advantages of better imaging resolution over polarized light microscopy, and could resolve collagen better than confocal microscopy alone [38]. In the past decade, SHG microscopy has become a powerful technique to image collagen fibrils in biological tissues with high contrast and specificity [38]. However, there are few studies using SHG examining the collagen fibrils in bone so far [39]. One unique feature of the MPM used in this study is that the fluorescence signals from TPEF were combined with SHG signals in a single microscope, which enabled us to directly link microcracking to collagen orientation.

\section{Results}

\section{Effects of Loading Orientation on Mechanical Properties and Strain Development}

Figure 2 shows typical load-displacement curves and representative shear stress-strain curves of the Iosipescu V-notch specimens at four different orientations with respect to the long bone axis (obtained from eight $0^{\circ}$, three $30^{\circ}$, five $60^{\circ}$ and four $90^{\circ}$ specimens). As large deformation causes optical decorrelation and makes DIC analyses invalid [40], stress-strain 
curves were plotted only before major cracks appeared (i.e. around the load peak). All stressstrain curves generally displayed an initial linear stage followed by a nonlinear stage.

The measured shear strength depended on specimen orientation (Table 1). The $0^{\circ}$ specimens showed the highest shear strength (49.9 $\mathrm{MPa} \pm 6.2 \mathrm{MPa})$, which is twice as high as that of the $60^{\circ}$ specimens $(22.7 \mathrm{MPa} \pm 2.5 \mathrm{MPa})$ (Table 1) $\underline{(p<0.05)}$. No statistically significant differences were observed among specimens oriented at $30^{\circ}, 60^{\circ}$ and $90^{\circ}(p>0.05)$. The shear moduli showed that $0^{\circ}$ specimens had the highest value (i.e. $4.0 \mathrm{GPa}$ ), while $30^{\circ}$ specimens had the lowest shear modulus (Table 1) although no statistical difference was found among the orientations. The ratio of transverse to longitudinal shear modulus $\left(\mathrm{G}_{0^{\circ}} / \mathrm{G}_{90^{\circ}}\right)$ obtained in the current study was 1.1, which is in agreement with a previous torsion study [41].

According to the pQCT measurements, there was no significant difference in bone density among the specimens $(p>0.05)$. However, we noted a larger standard deviation in $30^{\circ}$ specimens (Table 1).
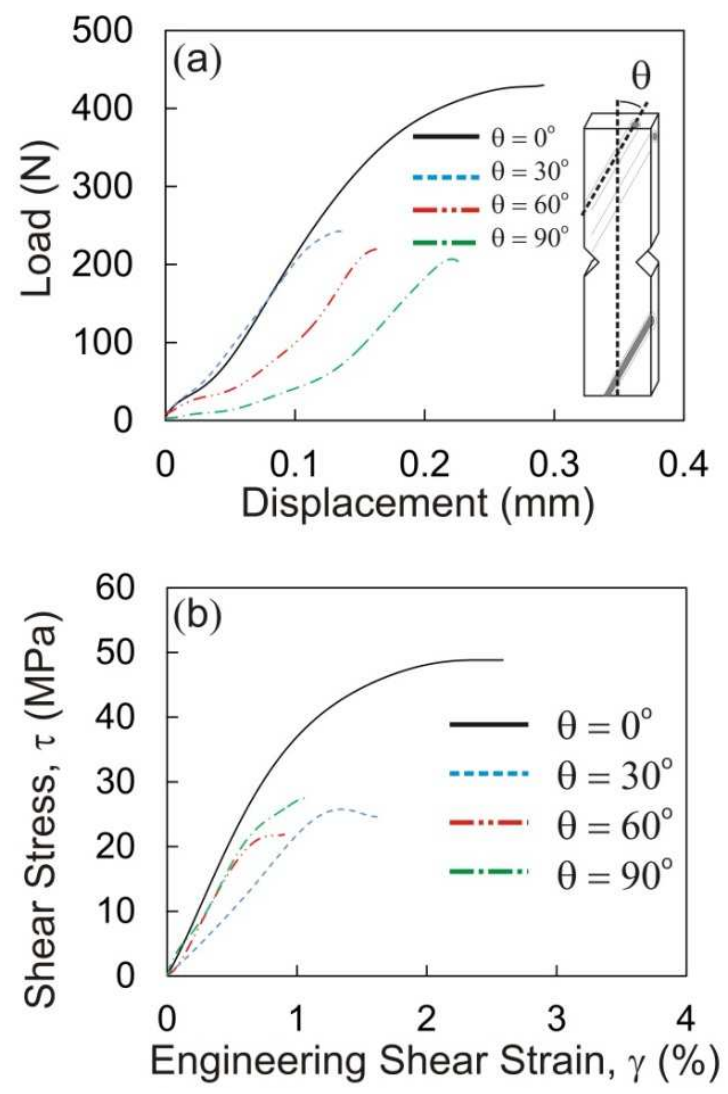

Fig. 2 Representative load-displacement curves (a) and stress-strain curves (b), for different specimens $\left(0^{\circ}, 30^{\circ}, 60^{\circ}\right.$ and $90^{\circ}$ ) tested by Iosipescu in-plane shear. Shear stress calculation following ASTM standard (D5379/D5379M) ( $\tau=\frac{P}{A}$, where $p$ is the loading force and $A$ is the cross-sectional area between two notch tips), and engineering shear 
strain obtained from DIC $\left(\gamma=2 \times \varepsilon_{x y}\right.$, where shear strain $\varepsilon_{x y}$ is the average value of the displacement gradients $e_{x y}$ and $e_{y x}$, which is the shear component on the y plane along the $\mathrm{x}$ direction and on the $\mathrm{x}$ plane along the $\mathrm{y}$ direction, respectively). Transverse shear specimens (i.e. $0^{\circ}$ ) showed a significantly higher failure strength and longer inelastic deformation process.

The in-plane strain triaxiality ratio $\left(\mathrm{TR}^{*}\right)$ before large inelastic deformation appeared was obtained from the specimens' gauge section using DIC analysis. The absolute value remained within a range of 0 to 0.12 , significantly less than $1 / 3$. This suggests the tested section was mainly under shear strain. The principal normal strain angle (i.e. between principal normal strain and horizontal direction which is perpendicular to specimens' long axis) for the gauge sections was $42.4^{\circ} \pm 2.8^{\circ}$. This represents fair agreement with the theoretical value for pure shear (a principal angle of $45^{\circ}$ should be expected). This further supports that the gauge section was primarily governed by shear rather than compression or tension.

Table 1. Bone mineral density, shear mechanical properties and fracture features of human cortical bone under shear at different orientations.

\begin{tabular}{|c|c|c|c|c|}
\hline Specimen Orientation & 0 degree & 30 degree & 60 degree & 90 degree \\
\hline Specimen Density $\left(\mathrm{g} / \mathrm{cm}^{3}\right)$ & $\begin{array}{l}1276.0 \pm \\
38.4\end{array}$ & $\begin{array}{l}1193.2 \pm \\
438.2\end{array}$ & $\begin{array}{c}1218.3 \pm \\
118.8\end{array}$ & $\begin{array}{c}1272.7 \pm \\
58.9\end{array}$ \\
\hline Shear Modulus [GPa] & $4.0 \pm 0.4$ & $2.9 \pm 1.6$ & $3.5 \pm 0.8$ & $3.5 \pm 0.5$ \\
\hline Shear Strength [MPa] & $49.9 \pm 6.2$ & $31.2 \pm 8.4$ & $22.7 \pm 2.5$ & $28.9 \pm 6.2$ \\
\hline $\begin{array}{c}\text { Angle of Shear Strain Band vs. Long } \\
\text { Bone Axis* }\left(^{(}\right)\end{array}$ & $14.0 \pm 6.7$ & $14.2 \pm 6.7$ & $11.8 \pm 3.4$ & $18.5 \pm 7.0$ \\
\hline $\begin{array}{c}\text { Angle of Major Crack Initiation vs. } \\
\text { Long Axes of Osteons }\left({ }^{\circ}\right)\end{array}$ & $\begin{array}{l}36.6 \pm \\
10.5\end{array}$ & $1.8 \pm 1.5$ & $2.5 \pm 0.5$ & $4.0 \pm 2.4$ \\
\hline $\begin{array}{c}\text { Major Crack Deflection by Cement } \\
\text { Line }(\%)\end{array}$ & N/A & $7.0 \pm 2.4$ & $11.8 \pm 8.3$ & $4.0 \pm 6.9$ \\
\hline $\begin{array}{c}\text { Major Crack Deflection by Lamellae } \\
\qquad(\%)\end{array}$ & N/A & $70.5 \pm 4.7$ & $65.7 \pm 10.1$ & $\begin{array}{c}55.9 \pm \\
17.0\end{array}$ \\
\hline $\begin{array}{c}\text { Major Crack Going through Haversian } \\
\text { Canals }(\%)\end{array}$ & N/A & $22.4 \pm 3.0$ & $22.5 \pm 14.2$ & $\begin{array}{c}40.0 \pm \\
18.3\end{array}$ \\
\hline
\end{tabular}


* The accuracy of long axis of osteons measurement under DIC is limited by resolution, thus the ideal long bone axis direction was used for comparison.

We used strain mapping, and showed that elastic deformation on the specimens was relatively homogeneous. In contrast, one or more high-deformation bands developed across the specimen during the inelastic stage (Figure 3). The deformation bands were $100 \mu \mathrm{m} \sim 150 \mu \mathrm{m}$ wide, and strain values were $5 \sim 7$ times larger than the average global strain. We noted the distinct directions of the developed deformation bands and their dependence on the specimen and osteons' orientations. In the $0^{\circ}$ specimens, the bands were generally aligned with the specimens' axis. The $90^{\circ}$ specimen showed bands that were more or less perpendicular to the axis. In the $30^{\circ}$ and $60^{\circ}$ specimens, bands were oblique, with a lower angle in the $60^{\circ}$ specimens. In general, shear strain concentration bands had a close association with long bone axis with an average deviation of $11.8^{\circ}$ to $18.5^{\circ}$ (Table 1 ). We also noted that there was a small deviation between the actual long bone axis (i.e. long axis of osteons) and the ideal orientation, which generally ranged from $8^{\circ}$ to $10^{\circ}$.

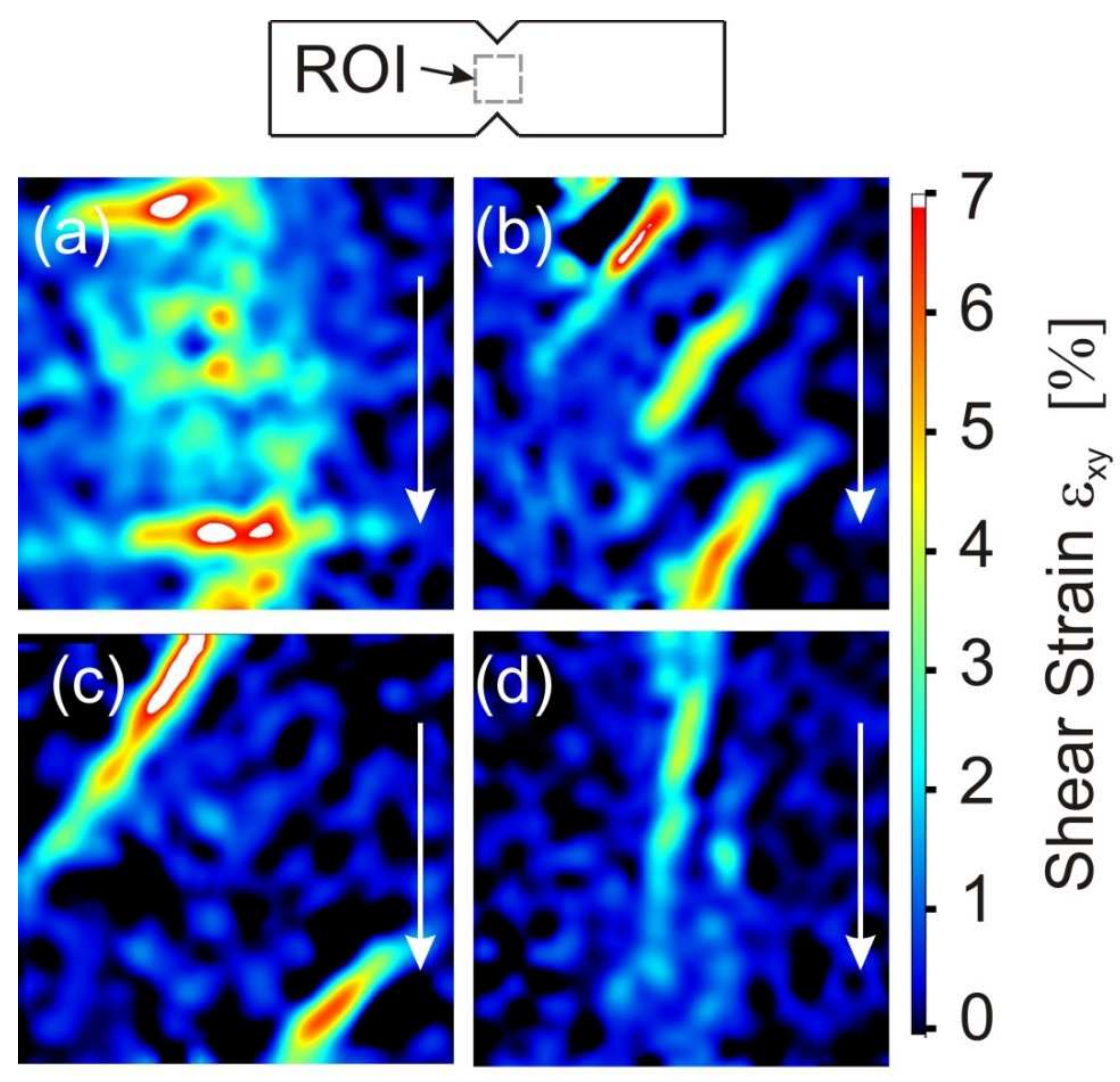

Fig. 3 Schematic diagram of region of interest (ROI) used for DIC shear strain analysis and the representative shear strain (i.e. $\varepsilon_{x y}$ ) distribution beyond yielding point, for specimens in each orientation: (a) $0^{\circ}$; (b) $30^{\circ}$; (c) $60^{\circ}$ and (d) 
$90^{\circ}$. The shear strain bands roughly follow the direction of the long bone, implying the role of bone's lamellar structure. Arrows indicate the shear loading direction.

\section{Macro-Scale Fracture Patterns}

Representative major fracture patterns for each orientation are provided in Figure 4. The cracking process strongly depended on the orientation of the specimens. Particularly, the angle between the crack initiation near the notches and the long axis of osteons was very small in $90^{\circ}$, $60^{\circ}$ and $30^{\circ}$ specimens, but dramatically increased to about 36 degree in the $0^{\circ}$ specimens (Table 1). In the $90^{\circ}$ specimens, where the loading direction coincided with the overall orientation of the osteons, the crack extended along the long axes of osteons in a relatively straight path (Figure $4 \mathrm{~d}$ ). For the $30^{\circ}$ and $60^{\circ}$ specimens, the cracks mostly followed the osteons, but were deflected more frequently than that in the $90^{\circ}$ specimens (Figure $4 \mathrm{~b}$ and c). When the orientation of the osteons was mainly perpendicular to the loading direction (i.e. $0^{\circ}$ specimens), specimens fractured in an off-axial two-path mode (Figure 4a). From the fracture pattern we observed for each specimen and using data recorded by high speed video, we concluded that the crack initiated at the upper or lower notch near the notch root and propagated along a direction that largely deviated from and tended to veer towards back to the osteons' long axis.
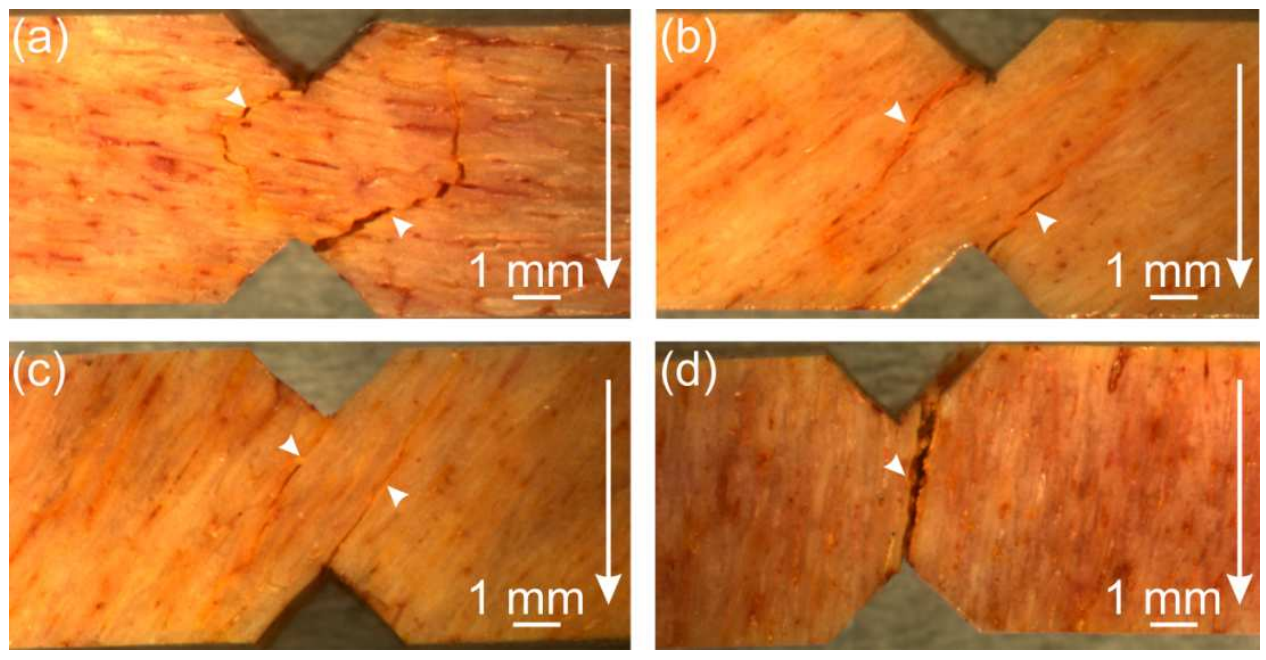

Fig. 4 Typical major fracture patterns under a stereo microscope. Of interest is the relatively large crack intiation angle vs. long axis of osteons, and the multiple crack deflections observed in $0^{\circ}$ specimen (a). $30^{\circ}$ (b), $60^{\circ}$ (c) and $90^{\circ}$ (d) specimens have comparatively flat fracture routes. Arrow heads point at the fracture planes. Shear loading direction is indicated by the vertical arrow. 
We assessed the interaction between the main fracture and the osteonal structure using transverse sections that were perpendicular to the long axes of osteons. When the main crack reached an osteon, it would: 1) be deflected by the cement line and went around the osteon (Figure 5a); 2) propagate into the osteon for a few layers of lamellae before being deflected by the lamellar structure: following a circular crack path, and exiting the osteon (Figure 5b); or 3) pass through the central Haversian canal without being deflected (Figure 5c). Of all the osteons we observed (more than 70 osteons for each $30^{\circ}, 60^{\circ}$ and $90^{\circ}$ orientation) at the major fracture paths, approximately $4.0 \%$ to $11.8 \%$ showed crack deflection at cement lines (Table 1 ). The lamellar structure, in the present study, seemed to play a major role in interfering with the cracking path. Over $\sim 50 \%$ of the observed osteons showed deflection at the lamellae (Table 1). However, lamellae's ability to deflect incoming cracks is a combined result of the direction of the incoming crack, the local lamellar orientation, and the loading direction. For the $0^{\circ}$ specimens, we observed that initiation near the notch sites on longitudinal sections were also influenced by the lamellar structure. Out of the four observed areas (i.e. at notch tips with clear lamellar structures), three showed cracks that followed the lamellar boundaries. Crack deflection at cement line was also observed, as shown in Figure 5d.
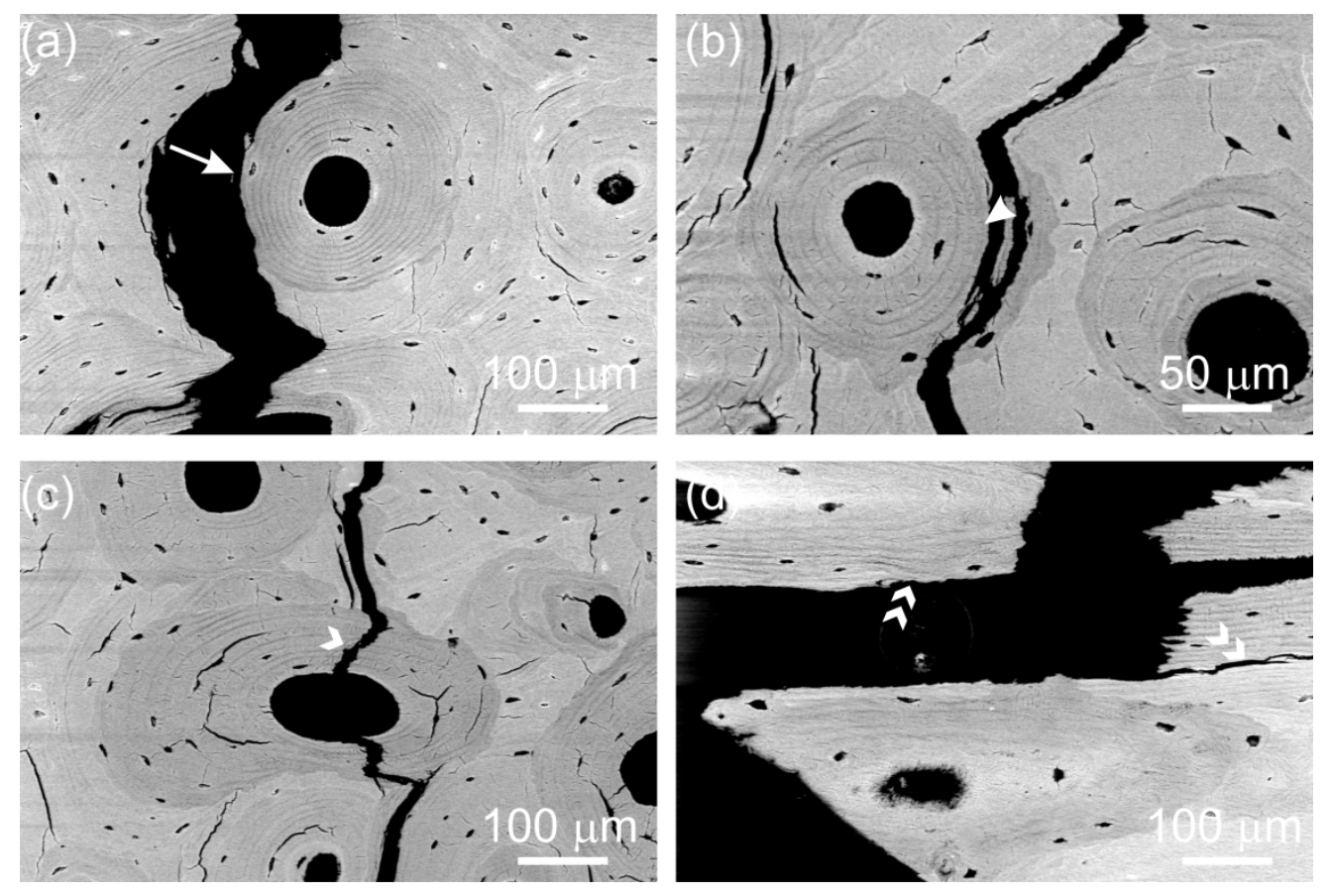

Fig. 5 SEM micrographs showing major fracture interacting with osteons. (a-c) represent three scenarios observed on transverse sections (plane perpendicular to long axes of osteons): (a) crack was deflected by the cement line and 
went around the osteon (arrow); (b) crack was deflected by bone lamellae inside the osteon, resulting in a circular crack path (arrow head); (c) crack went through the Haversian canal with minimum deflection (chevron arrow); (d) initiation crack was deflected along the cement line (double arrows) as observed from longitudinal section for $0^{\circ}$ specimen.

\section{Microcracking and Unique Arc-Shaped Microcracks}

We observed extensive damage when the tested specimens were sectioned perpendicular to the long axes of osteons (Figure 6). The intensity and distribution of microcracks stained by fluorescein depended on specimen orientation. In the $0^{\circ}$ specimens, damage over the entire section surface was homogeneously distributed (Figure 6a). On the contrary, $30^{\circ}, 60^{\circ}$ (Figure 6b) and $90^{\circ}$ specimens displayed damage that was localized near the main fractures.

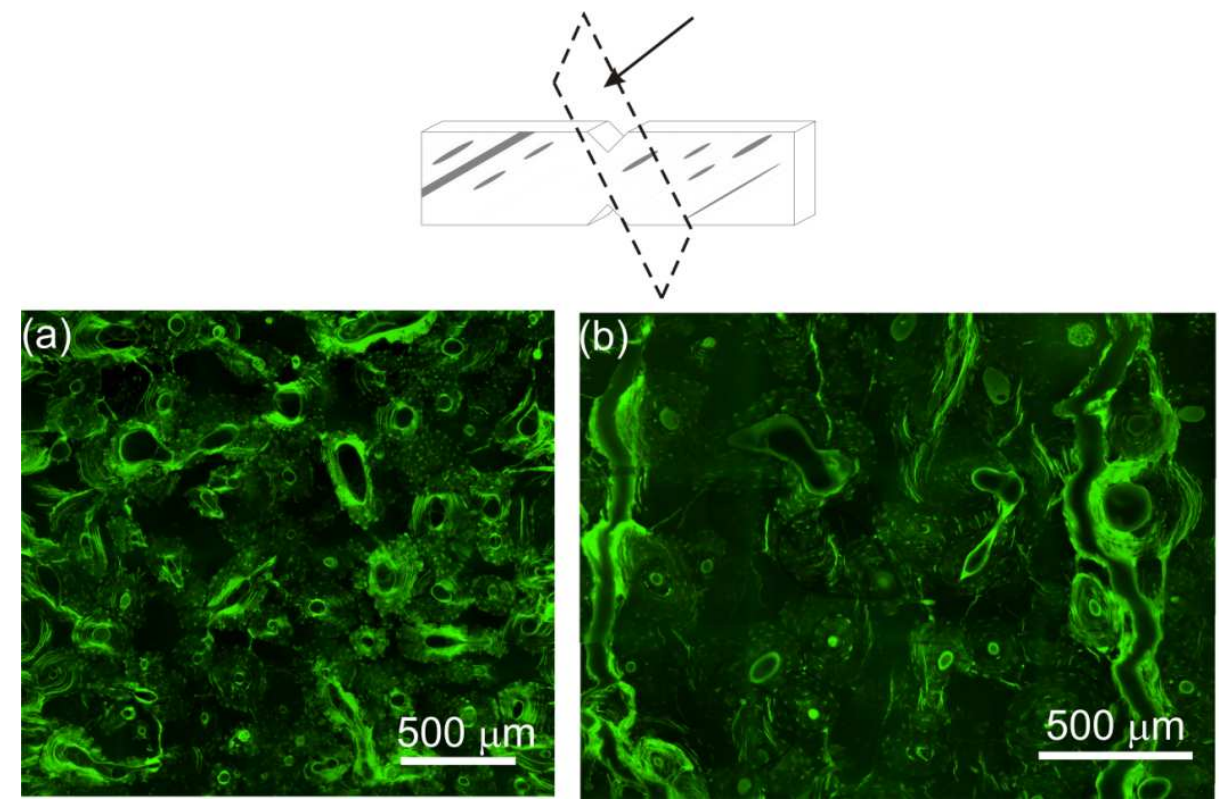

Fig. 6 LSCM images showing microcracks distribution in the gauge sections. The obtained cross-sections were all cut perpendicular to the osteons' long axes (indicated by grey lines) for analysis as shown in the schematic illustration. (a) and (b) show the representative damage pattern for $0^{\circ}$ and $60^{\circ}$ specimens. The $0^{\circ}$ specimen showed uniformly distributed damage while damage tended to localize around the major cracks in $60^{\circ}$ specimen.

At higher magnification, we identified six types of microcracks based on their location and shape: 1) osteonal peripheral arc-shaped cracks (Figure 7b) that were usually located away from the Haversian canals and followed the general contour of the lamellar boundaries; 2) osteonal tensile cracks; 3) osteonal radial cracks (supporting material: Figure S3d); 4) cement line cracks (supporting material: Figure S3b); 5) interstitial arc-shaped cracks; 6) interstitial 
tensile cracks. Arc-shaped cracks clearly differed from compressive cross-hatched cracks and tensile wavy cracks in that they were linear and long microcracks often constrained within a lamella without crossing its boundaries. The 3D imaging with LSCM (Figure 7b) showed that arc-shaped cracks had a "linear" appearance through the depth (z-plane), and stayed within the same lamellae. There were subtle variations in sub-micron morphology among the arc-shaped cracks: some appeared to be smoother, while others had regular crossed-lines or feather-like fine branches. Figure 8a shows the frequency of osteons showing each type of crack. Only the osteons at early stage of damage (less than ten microcracks with a length longer than $10 \mu \mathrm{m}$ ) were counted. Late stage deformation involved a more complicated stress state that might obscure the nature of the microcracking process. The final quantification depends on how many types of crack each osteon had, rather than the exact crack number. It is clear that the majority (over $80 \%$ ) of osteons at early stage of damage showed arc-shaped cracks.
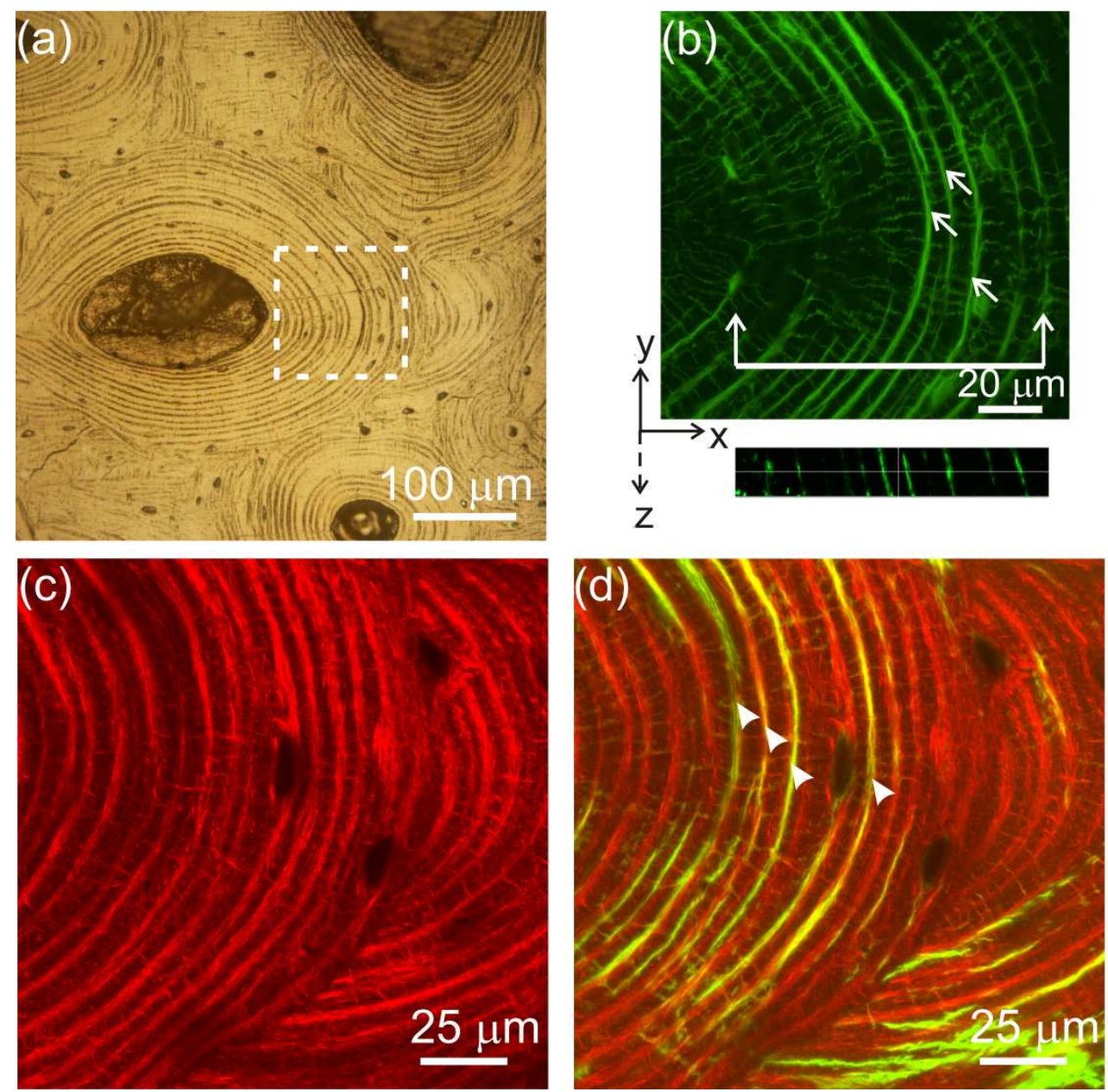

Fig. 7 Unique microcracking pattern under shear: (a) Optical Microscopic (OM) image showing the lamellar structure (i.e. $0^{\circ}$ ); (b) High resolution Laser Scanning Confocal Microscope (LSCM) image taken from the right side 
of the central osteon (dotted line) in (a). a unique peripheral arc-shaped pattern (arrows) is located at the peripheral zone of the osteon. They appear as linear microcracks on the z-plane ("cut views" at locations shown by white lines); (c) High resolution Second Harmonic Generation (SHG) image taken from the right bottom corner in (b) showing the osteon consisting of bright and dark lamellar layers. Note that in the bright layer, the collagen fibrils are mainly lying circumferential to the long axes of osteons, while the collagens are mostly align along the long axes of osteons in the dark layer; (d) Combined SHG and Two-Photon Excitation Fluorescence (TPEF) directly associating arc-shaped microcracks (arrow heads) with osteonal lamellae.

When we used LSCM to examine longitudinal surfaces, we did not see extensive damage at low magnification compared with the transverse sectional analysis. The longitudinal faces grounded and polished to the center of the three $0^{\circ}$ specimens showed similar sparse-distributed damage. However, we observed localized long and straight (linear) microcracks within bone lamellae at higher magnifications, corresponding to the z-plane view when we used 3D scanning on the transverse sections (Figure 7b).

\section{Relation between Microcracks and Bone Lamellae}

Arc-shaped microcracks were the dominant crack type. Therefore, we further analyzed the location of these microcracks in terms of lamellar structure inside the osteons. We defined five potential locations: P1 - at Haversian canal, P2 - inner third of the osteonal wall, P3 - middle third of the osteonal wall, P4 - outer third of osteonal wall and P5 - at cement line. We present the occurrence frequency of microcracking at these locations in Figure 8b. Briefly, over $70 \%$ of arc-shaped microcracks were located in the outer third of osteonal wall (i.e. peripheral layers of the osteonal wall).

The preferred orientation of collagen fibrils in each bone lamella can be assessed using SHG. The SHG image of a typical osteon is of alternating thin and thick lamellae reflecting different SHG signal intensities (Figure 7c). On a transvers section, collagen fibrils in the "bright" layers are oriented circumferentially around the Haversian canal, while those in the "dark" layers are preferentially aligned with the long axis of the osteon [42, 43]. TPEF image provides information about microcracking similar to what we obtained using LSCM (Figure 7d, green signal). The SHG image of collagen fibrils overlaid with the TPEF image of stained microcracks enabled us to directly relate shear microcracks with the different osteonal lamellae (Figure 7d). The arc-shaped cracks' association with lamellae of the early-damaged osteons showed variations among different orientations: $0^{\circ}, 30^{\circ}$ and $90^{\circ}$ specimens showed the 
confinement mainly in "bright" lamellae, while $60^{\circ}$ specimens had more cracks in the "dark" lamellae (Figure 8b).
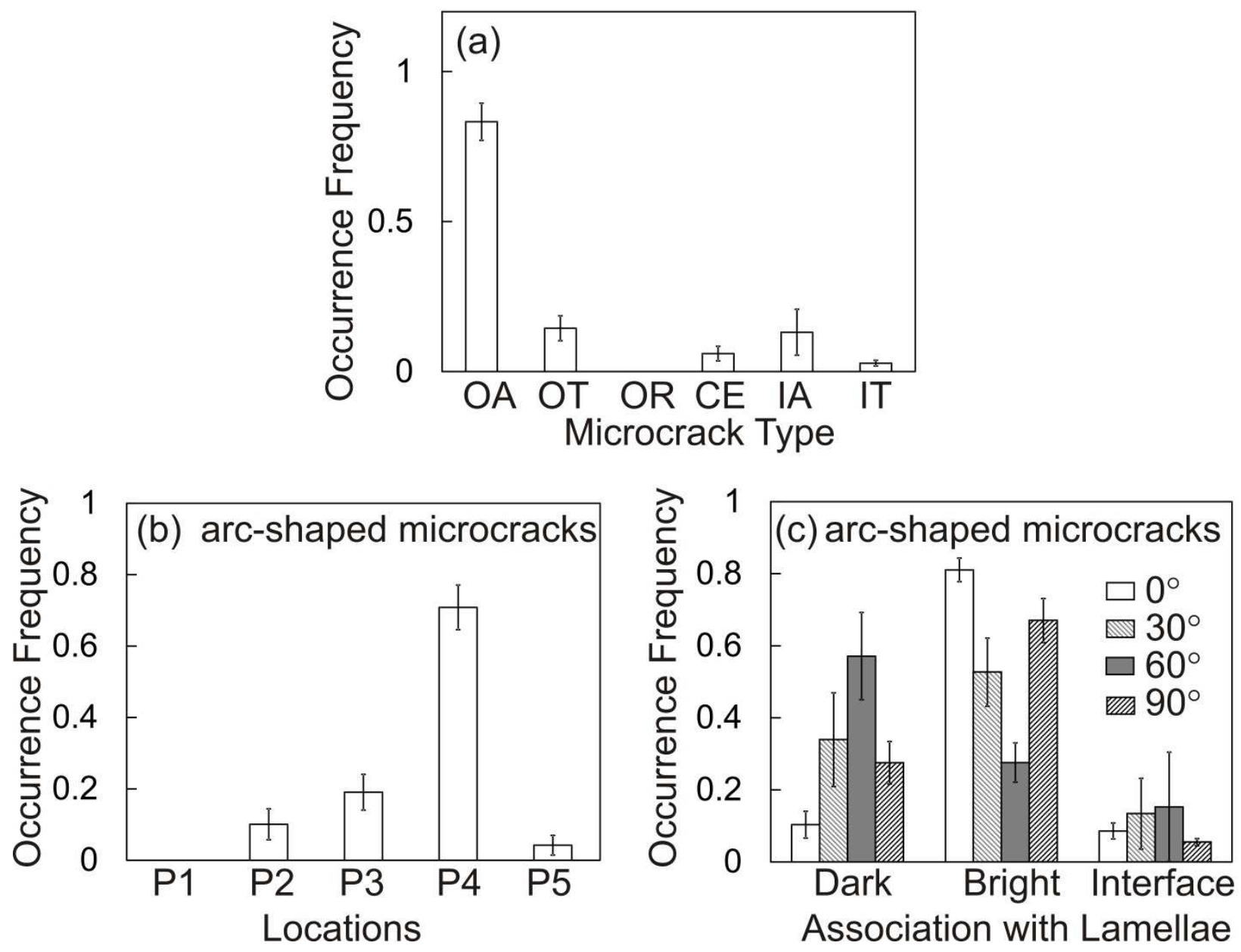

Fig. 8 Quantitative analysis on the microcracks created under shear. More than forty osteons from each orientation at early stage of damage were analyzed. (a) summary of occurrence frequency of six types of microcracks: osteonal arc-shaped (OA); osteonal tensile (OT); osteonal radial (OR); cement line (CE); interstitial arc-shaped (IA) and interstitial tensile (IT); (b) frequency distribution of the location of arc-shaped microcracks initiation. The osteon was divided into five locations: at the Haversian canal (P1); inner third of the osteonal wall (P2); middle third of the osteonal wall (P3); outer third of the osteonal wall (P4) and at the cement line (P5). (c) arc-shaped microcracks' association with lamellar structure (i.e. "bright"/“dark" lamellae and interlamellar area).

\section{Discussion}

This study demonstrates that human cortical bone under shear is anisotropic in its deformation and fracture process. Accompanying the inelastic deformation is a unique arcshaped microcracking pattern. High resolution combined analyses using second harmonic 
generation and two-photon excitation fluorescence clearly illustrated the important role for Haversian lamellae in the microcracking process.

\section{The Anisotropic Shear Behavior and the Structural Effects on Fracture}

That mechanical properties of cortical bone depend on orientation is well supported when loading in tension [44, 45] and compression [7]. Fewer studies have focused on the role of shear stress on bone's yield and ultimate failure mechanics. Turner et al. [20] reported Iosipescu shear strength against the long bone axis to be 65.3 $\mathrm{MPa} \pm 2.5 \mathrm{MPa}$, and Winwood et al. [28] reported that along the long bone axis to be $15 \mathrm{MPa}-40 \mathrm{MPa}$. We evaluated four different shear angles and confirmed that shear properties of human cortical bone are anisotropic. Shear strength and modulus in the transverse direction (shearing perpendicular to osteons' long axes in $0^{\circ}$

specimens) were higher than that at other orientations (Table 1), and the magnitudes were in reasonable concordance with those reported by Winwood [28]. The $0^{\circ}$ specimens also demonstrated a much longer inelastic deformation stage (Figure 2b). Bone can be viewed as a nano-fiber composite with mineralized collagen fibrils predominantly organized along the general direction of the long bone axis. Therefore, mechanical anisotropy may be explained using mechanisms that govern the mechanical anisotropy of engineering fiber reinforced matrix composites [3, 46-49]. Similar to fiber composites, inter-fibril shear damage or cracks may occur in bone during the inelastic deformation stage. When bone was sheared perpendicular to its long axis (i.e. $0^{\circ}$ specimens), mineralized collagen fibrils would bridge the inter-fibrous cracks and prevent them from developing into major fracture, leading to higher strength and strain to failure than noted in other specimen orientations. Since bone can be viewed as composite material, but exhibits more complicated structural organization at multiple length scales, the cracking and deformation processes would likely be more complicated than those in fiber composites. Research on bone's microstructural elements such as bone lamellae, cement lines, and Haversian canals is needed to further clarify their roles in physiologic and failure loading regimes.

The influence of the structural organization and anisotropy of osteons on the bone specimens' deformation process is evident in the shear strain patterns developed at the inelastic deformation stage. We applied DIC analysis, and revealed localized shear deformation bands at the inelastic stage. Interestingly, the strain bands were more or less aligned with the bone's long axis for all specimen orientations (Figure 3 and Table 1). This suggests a strong structural effect 
on the deformation process. This localization of shear strain directly leads to similar fracture patterns for $30^{\circ}, 60^{\circ}$ and $90^{\circ}$ specimens, which are roughly along the bone's long axis (Figure 4 b-d and Table 1). The shear bands in $0^{\circ}$ specimens could not propagate beyond the gauge section because of a less favorable stress state (the osteons may have acted as "crack arrestors"), and the fractures eventually developed at a larger angle (Figure 4a and Table 1), due to the principal stress in tension.

\section{Shear-induced Microcracks: The Unique Microcracking Pattern}

Previous studies demonstrated the importance of microcracking patterns as the basis for understanding bone's deformation and fracture processes [4-7, 50]. Compared with tension and compression, studies on shear-induced microcracking have been very limited. Forwood et al. [51] found cracks in rat tibia created under cyclic torsion running parallel to lamellae. Caler et al. [52] and George et al. [10] reported long shear microcracks (>300 $\mu \mathrm{m})$ under torsional loading with no or little diffuse damage. Vashishth et al. $[53,54]$ also described torsion created shear cracks as longitudinal linear cracks. Despite these reports, the nature of shear-induced microcraking at the lamellar and sub-lamellar levels remains largely unknown. We showed that arc-shaped microcracks locating at the peripheral zone of the osteons (Figure 7b) is the dominant pattern of shear-induced damage. They clearly differed from the reported densely distributed "wavy microcracks" for tension and the "cross-hatched" compression cracks. These differences suggest the influence of the sub-lamellar structure (i.e. the collagen fibril bundles organization) on the microcracking pattern. Sectional analysis under confocal microscopy (Figure 7b) found that the arc-shaped cracks propagated around the osteons and were confined to a single lamella.

The location where arc-shaped cracks initiated is worth noting. In the literature, the Haversian canal was frequently reported as a stress concentration site $[6,55]$. In human bone specimens subjected to transversal compression, Ebacher and Wang [7] found that Haversian canals are major strain concentration sites and are directly involved in crack initiation. However, the current study showed that the arc-shaped microcracks caused by shear happened at the peripheral zone of the osteons, closer to the cement line (Figure 8b). This unique microcracking process represents a different crack initiation and propagation mechanism. It appears that Haversian canals no longer play the dominant role under the shear condition. The exact nature of preferred microcracking at the peripheral zone of the osteons is not clear. Within the outer- 
layered lamellae, some intrinsically weak sites such as osteocyte lacunae and canaliculi could act as crack initiation sites [6, 22, 56, 57]. Indeed, in our LSCM examinations, we noted both osteocyte lacunae and canaliculi sites could act as part of the arc-shaped crack initiation.

\section{Role of Osteonal Lamellae, Collagen Fibrils, Cement Line and Lamellar Interface}

It has been hypothesized that the lamellar structure in osteonal bone plays a critical role in preventing catastrophic failures [3, 43, 58]. At the micro-level, the osteons are multilayered cylindrical structural units of mineralized fibrils arranged in lamellae around a central Haversian canal. Early studies using polarized light microscopy by Ascenzi and coworkers [16, 59] had suggested the existence of three types of osteons: bright (fibers in the lamellae transverse to the osteon axis), dark (fibers parallel to the axis) and alternating (alternating fiber orientations in adjacent layers), supporting the pioneer work by Gebhardt (1906). In the current study, the majority (about 85-90\%) of the osteons in our bone specimens had alternating bright and dark layers under the second harmonic generation microscope. On a transverse section, the bright lamellae were usually thin with collagen fibrils mainly lying circumferential to the long axes of osteons, while the dark lamellae were often thick with collagen fibrils mostly aligning along the long axes (Fig. 7). The SHG contrast matches that of alternate osteons under polarized light microscopy [60]. High resolution TEM studies by Weiner and coworkers found each pair of the alternating layers consisted of five successive layers of parallel fibrils oriented progressively every $30^{\circ}$, forming a rotated plywood structure [37]. Based on the results from X-ray microdiffraction and confocal microcscopy, Ascenzi et al. [61] further refined their model. The collagen fibers in the dark layers predominantly form small angles with the osteonal longitudinal axis, while those in the bright layers are predominantly organized at large angles with the osteonal longitudinal axis, i.e. $\mathbf{\pm 4 5}$ with respect to the osteon long axis with smaller amount of fibers perpendicular to the osteon axis. Recent work by Reznikov et al. on the 3D structure using dual-beam microscopy [62] showed that each pair of alternating layers had arrays of collagen fibrils organized into cylindrical rods and these in turn were aligned into higher order plywood $\underline{\text { structures with angles of } 45^{\circ}-80^{\circ} \text { between adjacent layers. Disordered collagen fibrils were also }}$ present surrounding the ordered structural motif. Many studies investigated the contribution of fibril organization to macroscopic mechanical properties [63-65]. Ascenzi and coworkers studied experimentally the role that collagen fiber orientation plays in the mechanical properties of 
single osteons under various loading conditions including tension [59, 66, 67], compression [16], torsion [15] and bending [68]. While those studies clearly demonstrated the effects of collagen fiber at a single osteonal level, directly relating collagen fibrillar organization to microcracking behavior at the tissue level remains a challenge. One innovative part of our study was that we successfully showed the relationship between lamellar structure and microcracking through the layered SHG and TPEF. Under the SHG imaging, alternating bright and dark lamellar layers could be clearly seen (Figure 7c). Simultaneously acquired TPEF signals provide microcracks information of the same region (Figure 7d, green signal). Subsequent quantitative analysis on the arc-shaped microcracks in $0^{\circ}, 30^{\circ}$ and $90^{\circ}$ specimens showed that they were preferentially located in the "bright" lamellae (Figure 8c), in which collagen fibrils are aligned circumferential to the long axes of osteons. In previous study on compression-induced shear cracking of osteonal bone [22], we also observed a similar preferential microcracking in a specific layer of the bone lamellae, although with no direct information on collagen organization. The observed phenomenon can be qualitatively explained using known theory in fiber composites. For $0^{\circ}$ and $90^{\circ}$ specimens, the circumferential fibrils for the "bright" lamellae would always have a portion of collagen fibrils perpendicular to both components of the shear stress, while the longitudinal fibrils in the "dark" lamellae would always be parallel to one of the shear components. The relation between fiber orientation and stress is very similar to the "interlaminar shear" described by Evans and Zok on two-dimensional ceramic matrix composites [47]. Inter-fibrillar cracks could evolve without significant interaction with the collagen fibrils in the "bright" lamellae, while in the "dark" lamellae, crack development would be impeded by surrounding collagen fibrils.

Cement lines, outlining secondary osteons, are frequently reported as points of weakness $[69,70]$. We also observed cement lines arresting and deflecting incoming cracks. However, cement line cracks only accounted for $\sim 6 \%$ of the early stage developed microcracks, significantly less frequent than intralamellar microcracking (Figure 8a). Additionally, less than $10 \%$ of the osteons at the main fracture sites had incoming cracks deflected at the cement lines (Table 1). Therefore, at least for shear induced deformation and fracture, a role for the cement lines is not as central as a role for bone lamellae.

The inter-lamellae or lamellar interface, characterized as a transition zone between bone lamellae, is considered as a weak interface where debonding occurs under tensile force [71]. 
Diab et al. [72] and Jepsen et al. [18] also reported that the lamellar interface damage is the primary cracking site under torsion. Although we observed arc-shaped cracks located near the lamellar interface, the majority of microcracks were confined within the lamellar layer, i.e. intralamellar cracks (Figure 8c). This was in agreement with previous studies [7, 22]. Therefore, our results support the conclusion that intralamellar microcracking is an important mechanism in post-yield deformation of human cortical bone subjected to shear.

Finally, our results are limited to small and unbalanced bone specimen sizes. The specimens are also limited to aged group, and the medial quadrant from femoral mid-shaft. Aging has been shown to adversely affect the elastic and ultimate properties of human cortical bone [73]. Studies have also shown that collagen orientation, degree of calcification vary across the quadrants and along the shaft $[74,75]$. Additionally, the osteon population density and osteon size may also vary due to the different rate of cortical bone turnover and biomechanical loadings [76]. Lamellar thickness on a transverse section is not always an alternating larger and smaller value; the completeness and thickness also vary among and within osteons [77, 78]. It would be interesting to see how changes in age and anatomical site affect the shear properties, the microcracking and fracture process.

\section{Conclusions}

We extended the current literature in two important ways. First, we illustrated how arcshaped microcracks resulted under shear loading clearly differed from tension and compression cracks by developing peripherally following the osteonal contour without coalescing. Second, we combined SHG with TPEF observations of human cortical bone under shear to directly relate microcracking to the lamellar structures of osteonal bone. This pattern was predominantly correlated with the "bright" layers, where mineralized collagen fibrils are mostly circumferential to the long axes of osteons, when shearing perpendicular to the osteons' long axes. Our findings provide new evidence regarding the structural importance of microcracking at the sub-lamellar level of mineralized collagen fibrils, and represent a next step toward better understanding on how microcracks are formed at the ultrastructural level. 


\section{Acknowledgements}

This study was supported by the Canadian Institutes of Health Research. Rizhi Wang is incumbent of the Canada Research Chair in Biomaterials and Tengteng Tang of the University Graduate Fellowship from UBC. We are thankful to Dr. Danmei Liu from the Centre for Hip Health and Mobility (CHHM) for discussions and assistance in the experiments and statistics. We are also thankful to the UBC Life Science Institute and UBC Department of Dentistry imaging facility for the use of their LSCM, to Dr. Kevin Hodgson at UBC BioImaging for the support in MPM imaging, and to Dr. Ya Shen and Dr. Zhejun Wang at UBC Dentistry for the kind assistance in Imaris 3D reconstruction. 


\section{Reference}

[1] Sterling RS. Gender and race/ethnicity differences in hip fracture incidence, morbidity, mortality, and function. Clinical orthopaedics and related research 2011;469: 1913-1918.

[2] Abrahamsen B, van Staa T, Ariely R, Olson M, Cooper C. Excess mortality following hip fracture: a systematic epidemiological review. Osteoporosis international : a journal established as result of cooperation between the European Foundation for Osteoporosis and the National Osteoporosis Foundation of the USA 2009;20: 1633-1650.

[3] Wang R, Gupta HS. Deformation and fracture mechanisms of bone and nacre. Annual Review of Materials Research 2011;41: 12.1-12.33 (in press).

[4] Zioupos P, Currey JD. The extent of microcracking and the morphology of microcracks in damaged bone. Journal of Materials Science 1994;29: 978-986.

[5] Boyce TM, Fyhrie DP, Glotkowski MC, Radin EL, Schaffler MB. Damage type and strain mode associations in human compact bone bending fatigue. Journal of orthopaedic research : official publication of the Orthopaedic Research Society 1998;16: 322-329.

[6] Reilly GC, Currey JD. The development of microcracking and failure in bone depends on the loading mode to which it is adapted. The Journal of experimental biology 1999;202: 543-552. [7] Ebacher V, Wang R. A unique microcracking process associated with the inelastic deformation of Haversian bone. Advanced Functional Materials 2009;19: 57-66.

[8] Mercer C, He MY, Wang R, Evans AG. Mechanisms governing the inelastic deformation of cortical bone and application to trabecular bone. Acta Biomaterialia 2006;2: 59-68.

[9] Ebacher V, Tang C, McKay H, Oxland TR, Guy P, Wang R. Strain redistribution and cracking behavior of human bone during bending. Bone 2007;40: 1265-1275.

[10] George WT, Vashishth D. Damage mechanisms and failure modes of cortical bone under components of physiological loading. Journal of orthopaedic research : official publication of the Orthopaedic Research Society 2005;23: 1047-1053.

[11] Taylor D, O'Reilly P, Vallet L, Lee TC. The fatigue strength of compact bone in torsion. Journal of Biomechanics 2003;36: 1103-1109.

[12] Zimmermann EA, Launey ME, Ritchie RO. The significance of crack-resistance curves to the mixed-mode fracture toughness of human cortical bone. Biomaterials 2010;31: 5297-5305. 
[13] Mayhew PM, Thomas CD, Clement JG, Loveridge N, Beck TJ, Bonfield W, Burgoyne CJ, Reeve J. Relation between age, femoral neck cortical stability, and hip fracture risk. The Lancet 2005;366: 129-135.

[14] Turner CH. The biomechanics of hip fracture. The Lancet 2005;366: 98-99.

[15] Ascenzi A, Bonucci E. The shearing properties of single osteons. The Anatomical Record 1972;172: 499-510.

[16] Ascenzi A, Bonucci E. The compressive properties of single osteons. The Anatomical Record 1968;161: 377-391.

[17] Ascenzi A, Bonucci E, Simkin A. An approach to the mechanical properties of single osteonic lamellae. Journal of Biomechanics 1973;6: 227-235.

[18] Jepsen KJ, Davy DT, Krzypow DJ. The role of the lamellar interface during torsional yielding of human cortical bone. Journal of Biomechanics 1999;32: 303-310.

[19] Saha S. Longitudinal shear properties of human compact bone and its constituents, and the associated failure mechanisms. In: Springer Netherlands; 1977. p. 1798-1806.

[20] Turner CH, Wang T, Burr DB. Shear strength and fatigue properties of human cortical bone determined from pure shear tests. Calcified tissue international 2001;69: 373-378.

[21] Dong XN, Luo Q, Wang X. Progressive post-yield behavior of human cortical bone in shear. Bone 2013;53: 1-5.

[22] Ebacher V, Guy P, Oxland TR, Wang R. Sub-lamellar microcracking and roles of canaliculi in human cortical bone. Acta biomaterialia 2012;8: 1093-1100.

[23] Sheppard C, Gannaway J, Kompfner R, Walsh D. The scanning harmonic optical microscope. Quantum Electronics, IEEE Journal of 1977;13: 912-912.

[24] Rice WL, Kaplan DL, Georgakoudi I. - Two-Photon Microscopy for Non-Invasive, Quantitative Monitoring of Stem Cell Differentiation. In: - Public Library of Science. p. e10075.

[25] Campagnola P. Second Harmonic Generation Imaging Microscopy: Applications to Diseases Diagnostics. In: - Analytical Chemistry: - American Chemical Society; 2011. p. - 3224.

[26] Astm International WCPA. Standard Test Method for Shear Properties of Composite Materials by the V-Notched Beam Method. In; 1993.

[27] Lee TC, Mohsin S, Taylor D, Parkesh R, Gunnlaugsson T, O'Brien FJ, Giehl M, Gowin W. Detecting microdamage in bone. Journal of anatomy 2003;203: 161-172. 
[28] Winwood K, Zioupos P, Currey JD, Cotton JR, Taylor M. Strain patterns during tensile, compressive, and shear fatigue of human cortical bone and implications for bone biomechanics. Journal of Biomedical Materials Research Part A 2006;79A: 289-297.

[29] Adams DF, Walrath DE. Further development of the losipescu shear test method. Experimental Mechanics 1987;27: 113-119.

[30] Xavier JC, Garrido NM, Oliveira M, Morais JL, Camanho PP, Pierron F. A comparison between the Iosipescu and off-axis shear test methods for the characterization of Pinus Pinaster Ait. Composites Part A: Applied Science and Manufacturing 2004;35: 827-840.

[31] Pierron F. Saint-Venant Effects in the Iosipescu Specimen 1998;32: 1986-2015.

[32] Quinta Da Fonseca J, Mummery PM, Withers PJ. Full-field strain mapping by optical correlation of micrographs acquired during deformation. Journal of microscopy 2005;218: 9-21.

[33] Bao Y, Wierzbicki T. On fracture locus in the equivalent strain and stress triaxiality space. International Journal of Mechanical Sciences 2004;46: 81-98.

[34] Bai Y, Teng X, Wierzbicki T. On the Application of Stress Triaxiality Formula for Plane Strain Fracture Testing. Journal of Engineering Materials and Technology 2009;131: 021002021002.

[35] Daiyan H, Andreassen E, Grytten F, Osnes H, Gaarder R. Shear Testing of Polypropylene Materials Analysed by Digital Image Correlation and Numerical Simulations. In: Springer Boston; 2012. p. 1-15.

[36] Campagnola PJ, Loew LM. Second-harmonic imaging microscopy for visualizing biomolecular arrays in cells, tissues and organisms. Nat Biotech 2003;21: 1356-1360.

[37] Weiner S, Wagner HD. The material bone: Structure mechanical function relations. Annual review of materials science 1998;28: 271-298.

[38] Ambekar R, Chittenden M, Jasiuk I, Toussaint Jr KC. Quantitative second-harmonic generation microscopy for imaging porcine cortical bone: Comparison to SEM and its potential to investigate age-related changes. Bone 2012;50: 643-650.

[39] Sun X, McLamore E, Kishore V, Fites K, Slipchenko M, Porterfield DM, Akkus O. Mechanical Stretch Induced Calcium Efflux from Bone Matrix Stimulates Osteoblasts. Bone 2012;50: 581-591. 
[40] Shahar R, Zaslansky P, Barak M, Friesem AA, Currey JD, Weiner S. Anisotropic Poisson's ratio and compression modulus of cortical bone determined by speckle interferometry. Journal of Biomechanics 2007;40: 252-264.

[41] Neil Dong X, Edward Guo X. The dependence of transversely isotropic elasticity of human femoral cortical bone on porosity. Journal of Biomechanics 2004;37: 1281-1287.

[42] Kazanci M, Wagner HD, Manjubala NI, Gupta HS, Paschalis E, Roschger P, Fratzl P. Raman imaging of two orthogonal planes within cortical bone. Bone 2007;41: 456-461.

[43] Wagermaier W, Gupta HS, Gourrier A, Burghammer M, Roschger P, Fratzl P. Spiral twisting of fiber orientation inside bone lamellae. Biointerphases 2006;1: 1-5.

[44] Norman TL, Vashishth D, Burr DB. Fracture toughness of human bone under tension. Journal of Biomechanics 1995;28: 309-320.

[45] Fan Z, Swadener JG, Rho JY, Roy ME, Pharr GM. Anisotropic properties of human tibial cortical bone as measured by nanoindentation. Journal of orthopaedic research : official publication of the Orthopaedic Research Society 2002;20: 806-810.

[46] Broughton WR, Kumosa M, Hull D. Analysis of the Iosipescu shear test as applied to unidirectional carbon-fibre reinforced composites. Composites Science and Technology 1990;38: 299-325.

[47] Evans AG, Zok FW. The physics and mechanics of fiber-reinforced brittle matrix composites. Journal of Materials Science 1994;29: 3857-3896.

[48] BrÃ ndsted P, Heredia FE, Evans AG. In-plane Shear Properties of 2-D Ceramic Matrix Composites. Journal of the American Ceramic Society 1994;77: 2569-2574.

[49] Ascenzi A, Bonucci E. Mechanical similarities between alternate osteons and cross-ply laminates. Journal of Biomechanics 1976;9: 65-71.

[50] Vashishth D, Tanner KE, Bonfield W. Contribution, development and morphology of microcracking in cortical bone during crack propagation. Journal of Biomechanics 2000;33: 1169-1174.

[51] Forwood MR, Parker AW. Microdamage in response to repetitive torsional loading in the rat tibia. Calcified tissue international 1989;45: 47-53.

[52] Caler WE, Carter DR. Bone creep-fatigue damage accumulation. Journal of Biomechanics 1989;22: 625-635. 
[53] George WT, Vashishth D. Influence of phase angle between axial and torsional loadings on fatigue fractures of bone. In; 2005. p. 819.

[54] Vashishth D, Tanner KE, Bonfield W. Fatigue of cortical bone under combined axialtorsional loading. Journal of Orthopaedic Research 2001;19: 414-420.

[55] Carter DR, Hayes WC. Compact bone fatigue damage: a microscopic examination. Clinical orthopaedics and related research 1977;(127): 265-274.

[56] Nicolella DP, Moravits DE, Gale AM, Bonewald LF, Lankford J. Osteocyte lacunae tissue strain in cortical bone. Journal of Biomechanics 2006;39: 1735-1743.

[57] Ascenzi M-G, Gill J, Lomovtsev A. Orientation of collagen at the osteocyte lacunae in human secondary osteons. Journal of Biomechanics 2008;41: 3426-3435.

[58] Gupta HS, Stachewicz U, Wagermaier W, Roschger P, Wagner HD, Fratzl P. Mechanical modulation at the lamellar level in osteonal bone. Journal of Materials Research 2006;21: 19131921.

[59] Ascenzi A, Bonucci E. The tensile properties of single osteons. The Anatomical Record 1967;158: 375-386.

[60] Ascenzi MG, Roe AK. The osteon: the micromechanical unit of compact bone. Frontiers in Bioscience-Landmark 2012;17: 1551-1581.

[61] Ascenzi M-G, Ascenzi A, Benvenuti A, Burghammer M, Panzavolta S, Bigi A. Structural differences between "dark" and "bright" isolated human osteonic lamellae. Journal of structural biology 2003;141: 22-33.

[62] Reznikov N, Shahar R, Weiner S. Three-dimensional structure of human lamellar bone: The presence of two different materials and new insights into the hierarchical organization. Bone 2014;59: 93-104.

[63] Gao H, Ji B, Jager IL, Arzt E, Fratzl P. Materials become insensitive to flaws at nanoscale: lessons from nature. Proceedings of the National Academy of Sciences of the United States of America 2003;100: 5597-5600.

[64] Nalla RK, Kinney JH, Ritchie RO. Mechanistic fracture criteria for the failure of human cortical bone. Nat.Mater. 2003;2: 164-168.

[65] Peterlik H, Roschger P, Klaushofer K, Fratzl P. From brittle to ductile fracture of bone. Nature materials 2006;5: 52-55. 
[66] Ascenzi A - Bonucci E. THE ULTIMATE TENSILE STRENGTH OF SINGLE OSTEONS. Acta. Anat. 1964;58: 23.

[67] Ascenzi A, Benvenuti A, Bonucci E. The tensile properties of single osteonic lamellae: Technical problems and preliminary results. Journal of Biomechanics 1982;15: 29-37.

[68] Ascenzi A, Baschieri P, Benvenuti A. The bending properties of single osteons. Journal of Biomechanics 1990;23: 763-771.

[69] Martin RB, Burr DB. Structure, Function, and Adaptation of Compact Bone. New York: Raven Press; 1989.

[70] O'Brien FJ, Taylor D, Lee TC. Bone as a composite material: The role of osteons as barriers to crack growth in compact bone. In. [Guildford, England]: Elsevier; 2007. p. 10511056.

[71] Wasserman N, Brydges B, Searles S, Akkus O. In vivo linear microcracks of human femoral cortical bone remain parallel to osteons during aging. Bone 2008;43: 856-861.

[72] Diab T, Vashishth D. Morphology, localization and accumulation of in vivo microdamage in human cortical bone. Bone 2007;40: 612-618.

[73] Zioupos P, Currey JD. Changes in the stiffness, strength, and toughness of human cortical bone with age. Bone 1998;22: 57-66.

[74] Portigliatti-Barbos M, Carando S, Ascenzi A, Boyde A. On the structural symmetry of human femurs. Bone 1987;8: 165-169.

[75] Evans FG, Vincentelli R. Relations of the compressive properties of human cortical bone to histological structure and calcification. Journal of Biomechanics 1974;7: 1-10.

[76] Skedros JG, Keenan KE, Williams TJ, Kiser CJ. Secondary osteon size and collagen/lamellar organization (“osteon morphotypes") are not coupled, but potentially adapt independently for local strain mode or magnitude. Journal of structural biology 2013;181: 95107.

177] Ascenzi M-G, Lomovtsev A. Collagen orientation patterns in human secondary osteons, quantified in the radial direction by confocal microscopy. Journal of structural biology 2006;153: $\underline{14-30 .}$

[78] Ascenzi MG, Andreuzzi M Fau - Kabo JM, Kabo JM. Mathematical modeling of human secondary osteons. SCANNING 2004;26: 3. 


\section{Figure Captions}

Fig. 1 Schematic illustration of specimens prepared from human femora. Shear specimens were oriented at $0^{\circ}, 30^{\circ}, 60^{\circ}$ and $90^{\circ}$ with respect to the long axis of femora, which is indicated by the grey lines. A specimen was gripped in the custom made Wyoming Iosipescu fixture with the shear loading indicated by the arrow pointing at the top of the rod.

Fig. 2 Representative load-displacement curves (a) and stress-strain curves (b), for different specimens $\left(0^{\circ}, 30^{\circ}, 60^{\circ}\right.$ and $\left.90^{\circ}\right)$ tested by Iosipescu in-plane shear. Shear stress calculation following ASTM standard (D5379/D5379M) $\left(\tau=\frac{P}{A}\right.$, where $p$ is the loading force and $A$ is the cross-sectional area between two notch tips), and engineering shear strain obtained from DIC $\left(\gamma=2 \times \varepsilon_{x y}\right.$, where shear strain $\varepsilon_{x y}$ is the average value of the displacement gradients $e_{x y}$ and $e_{y x}$, which is the shear component on the y plane along the $\mathrm{x}$ direction and on the $\mathrm{x}$ plane along the y direction, respectively). Transverse shear specimens (i.e. $0^{\circ}$ ) showed a significantly higher failure strength and longer inelastic deformation process.

Fig. 3 Schematic diagram of region of interest (ROI) used for DIC shear strain analysis and the representative shear strain (i.e. $\varepsilon_{x y}$ ) distribution beyond yielding point, for specimens in each orientation: (a) $0^{\circ}$; (b) $30^{\circ}$; (c) $60^{\circ}$ and (d) $90^{\circ}$. The shear strain bands roughly follow the direction of the long bone, implying the role of bone's lamellar structure. Arrows indicate the shear loading direction.

Fig. 4 Typical major fracture patterns under a stereo microscope. Of interest is the relatively large crack initiation angle vs. long axis of osteons, and the multiple crack deflections observed in $0^{\circ}$ specimen (a). $30^{\circ}$ (b), $60^{\circ}$ (c) and $90^{\circ}$ (d) specimens have comparatively flat fracture routes. Arrow heads point at the fracture planes. Shear loading direction is indicated by the vertical arrow.

Fig. 5 SEM micrographs showing major fracture interacting with osteons. (a-c) represent three scenarios observed on transverse sections (plane perpendicular to long axes of osteons): (a) crack was deflected by the cement line and went around the osteon (arrow); (b) crack was deflected by bone lamellae inside the osteon, resulting in a circular crack path (arrow head); (c) crack went 
through the Haversian canal with minimum deflection (chevron arrow); (d) initiation crack was deflected along the cement line (double arrows) as observed from longitudinal section for $0^{\circ}$ specimen.

Fig. 6 LSCM images showing microcracks distribution in the gauge sections. The obtained cross-sections were all cut perpendicular to the osteons' long axes (indicated by grey lines) for analysis as shown in the schematic illustration. (a) and (b) show the representative damage pattern for $0^{\circ}$ and $60^{\circ}$ specimens. The $0^{\circ}$ specimen showed uniformly distributed damage while damage tended to localize around the major cracks in $60^{\circ}$ specimen.

Fig. 7 Unique microcracking pattern under shear: (a) Optical Microscopic (OM) image showing the lamellar structure (i.e. $0^{\circ}$ ); (b) High resolution Laser Scanning Confocal Microscope (LSCM) image taken from the right side of the central osteon (dotted line) in (a). a unique peripheral arcshaped pattern (arrows) is located at the peripheral zone of the osteon. They appear as linear microcracks on the z-plane ("cut views" at locations shown by white lines); (c) High resolution Second Harmonic Generation (SHG) image taken from the right bottom corner in (b) showing the osteon consisting of bright and dark lamellar layers. Note that in the bright layer, the collagen fibrils are mainly lying circumferential to the long axes of osteons, while the collagens are mostly align along the long axes of osteons in the dark layer; (d) Combined SHG and TwoPhoton Excitation Fluorescence (TPEF) directly associating arc-shaped microcracks (arrow heads) with osteonal lamellae.

Fig. 8 Quantitative analysis on the microcracks created under shear. More than forty osteons from each orientation at early stage of damage were analyzed. (a) summary of occurrence frequency of six types of microcracks: osteonal arc-shaped (OA); osteonal tensile (OT); osteonal radial (OR); cement line (CE); interstitial arc-shaped (IA) and interstitial tensile (IT); (b) frequency distribution of the location of arc-shaped microcracks initiation. The osteon was divided into five locations: at the Haversian canal (P1); inner third of the osteonal wall (P2); middle third of the osteonal wall (P3); outer third of the osteonal wall (P4) and at the cement line (P5). (c) arc-shaped microcracks' association with lamellar structure (i.e. "bright"/“dark" lamellae and interlamellar area). 
Table 1. Bone mineral density, shear mechanical properties and fracture features of human cortical bone under shear at different orientations.

\begin{tabular}{|c|c|c|c|c|}
\hline Specimen Orientation & 0 degree & 30 degree & 60 degree & 90 degree \\
\hline Specimen Density $\left(\mathrm{g} / \mathrm{cm}^{3}\right)$ & $\begin{array}{l}1276.0 \pm \\
38.4\end{array}$ & $\begin{array}{l}1193.2 \pm \\
438.2\end{array}$ & $\begin{array}{l}1218.3 \pm \\
118.8\end{array}$ & $\begin{array}{c}1272.7 \pm \\
58.9\end{array}$ \\
\hline Shear Modulus [GPa] & $4.0 \pm 0.4$ & $2.9 \pm 1.6$ & $3.5 \pm 0.8$ & $3.5 \pm 0.5$ \\
\hline Shear Strength [MPa] & $49.9 \pm 6.2$ & $31.2 \pm 8.4$ & $22.7 \pm 2.5$ & $28.9 \pm 6.2$ \\
\hline $\begin{array}{c}\text { Angle of Shear Strain Band vs. Long } \\
\text { Bone Axis* }\left(^{\circ}\right)\end{array}$ & $14.0 \pm 6.7$ & $14.2 \pm 6.7$ & $11.8 \pm 3.4$ & $18.5 \pm 7.0$ \\
\hline $\begin{array}{l}\text { Angle of Major Crack Initiation vs. } \\
\text { Long Axes of Osteons }\left(^{(}\right)\end{array}$ & $\begin{array}{l}36.6 \pm \\
10.5\end{array}$ & $1.8 \pm 1.5$ & $2.5 \pm 0.5$ & $4.0 \pm 2.4$ \\
\hline $\begin{array}{c}\text { Major Crack Deflection by Cement } \\
\text { Line }(\%)\end{array}$ & N/A & $7.0 \pm 2.4$ & $11.8 \pm 8.3$ & $4.0 \pm 6.9$ \\
\hline $\begin{array}{l}\text { Major Crack Deflection by Lamellae } \\
\qquad(\%)\end{array}$ & N/A & $70.5 \pm 4.7$ & $65.7 \pm 10.1$ & $\begin{array}{c}55.9 \pm \\
17.0\end{array}$ \\
\hline $\begin{array}{c}\text { Major Crack Going through Haversian } \\
\text { Canals }(\%)\end{array}$ & N/A & $22.4 \pm 3.0$ & $22.5 \pm 14.2$ & $\begin{array}{c}40.0 \pm \\
18.3\end{array}$ \\
\hline
\end{tabular}

* The accuracy of long axis of osteons measurement under DIC is limited by resolution, thus the ideal long bone axis direction was used for comparison. 